Georgian Mathematical Journal

1(1994), No. 2, 127-140

\title{
ON THE UNIQUENESS THEOREMS FOR THE EXTERNAL PROBLEMS OF THE COUPLE-STRESS THEORY OF ELASTICITY
}

\author{
T. BUCHUKURI AND T. GEGELIA
}

\begin{abstract}
A formula is obtained for the asymptotic representation of solutions of the basic equations of the couple-stress theory of elasticity. The formula is used in proving the uniqueness theorems of the external boundary value problems.
\end{abstract}

0. Let $\Omega^{+}$be a bounded domain in the three-dimensional Euclidean space $\mathbb{R}^{3}$, and $\Omega^{-}$a complement of $\Omega^{+}$to the entire space $\mathbb{R}^{3}: \Omega^{-}=\mathbb{R}^{3} \backslash \bar{\Omega}^{-}$. The boundary value problems formulated for the domain $\Omega^{-}$are called external. The uniqueness theorems for the external boundary value problems are valid only under some restrictions of the class of solutions at infinity ([1], [2]). These restrictions arose naturally from the Green formulas and consist in the requirement that both the solution and its derivatives vanish at infinity. The weakening of the restrictions is important from both the theoretical and the practical standpoints (for example, in constructing effective solutions). This question is discussed in the monograph [1] devoted specially to uniqueness theorems of the theory of elasticity.

In recent years new results have been obtained for the external static problems of the classical theory of elasticity [3]-[7]. In these works the authors have succeeded in weakening essentially the restrictions at infinity imposed on the class of solutions in which the uniqueness theorems are proved. The results were obtained by two different methods: in [4], [5] the proof was based on Korn's inequality, whereas in [3], [6], [7] use was made of the asymptotic representation of solutions in the neighborhood of an isolated singular point (in particular, in the neighborhood of the point at infinity). However, both methods were applied to the system of equations containing only derivatives of higher (second) order. The system of static equations of the classical elasticity theory is also such a system.

In this paper we show that the method of asymptotic representations of solutions in the neighborhood of an isolated singular point (see [3], [6], [7]) 
can be as well applied to systems of equations containing derivatives of both higher and lower orders. This is exemplified by the system of static equations of the couple-stress theory of elasticity for a homogeneous anisotropic medium containing derivatives of second order, as well as derivatives of first and zero orders. Here we have derived the asymptotic representation of the solution of the said system in the neighborhood of the point at infinity, which has enabled us to prove new uniqueness theorems for the external boundary value problems of the couple-stress theory of elasticity.

The derivation of asymptotic representations largely rests on the behavior of the fundamental solution of the considered system at infinity.

1. A homogeneous system of the couple-stress theory of a homogeneous anisotropic micropolar elastic medium is written in the form [2], [9]

$$
\begin{gathered}
c_{i j l k} \frac{\partial^{2} u_{k}}{\partial x_{j} \partial x_{l}}-c_{j i l m} \varepsilon_{k l m} \frac{\partial \omega_{k}}{\partial x_{j}}=0, \\
c_{j m l k} \varepsilon_{i j m} \frac{\partial u_{k}}{\partial x_{l}}+c_{j i l k}^{\prime} \frac{\partial^{2} \omega_{k}}{\partial x_{j} \partial x_{l}}-c_{j m l p} \varepsilon_{i j m} \varepsilon_{k l p} \omega_{k}=0, \quad i=1,2,3,
\end{gathered}
$$

where $u=\left(u_{1}, u_{2}, u_{3}\right)$ is the displacement vector, $\omega=\left(\omega_{1}, \omega_{2}, \omega_{3}\right)$ is the rotation vector, $\varepsilon_{i j k}$ is the Levi-Civita symbol, $c_{j i l k}, c_{j i l k}^{\prime}(i, j, l, k=1,2,3)$ are the elastic constants. Here and in what follows the repetition of the index in the product means summation over this index.

It is assumed that the elastic coefficients $c_{i j k l}$ and $c_{i j k l}^{\prime}$ satisfy the symmetry conditions

$$
c_{i j k l}=c_{k l i j}, \quad c_{i j k l}^{\prime}=c_{k l i j}^{\prime}
$$

and the energetic form is positive-definite

$$
c_{i j k l} \xi_{i j} \xi_{k l}+c_{i j k l}^{\prime} \eta_{i j} \eta_{k l}>0 \text { for } \xi_{i j} \xi_{i j}+\eta_{i j} \eta_{i j} \neq 0 .
$$

Let

$$
\begin{aligned}
& A\left(\partial_{x}\right) \equiv\left\|A_{i k}\left(\partial_{x}\right)\right\|_{6 \times 6}, \\
& A_{i k}\left(\partial_{x}\right) \equiv c_{j i l k} \frac{\partial^{2}}{\partial x_{j} \partial x_{l}}, \quad i, k=1,2,3 ; \\
& A_{i, k+3}\left(\partial_{x}\right) \equiv-c_{j i l m} \varepsilon_{k l m} \frac{\partial}{\partial x_{j}}, \quad i, k=1,2,3 ; \\
& A_{i+3, k}\left(\partial_{x}\right) \equiv c_{j m l k} \varepsilon_{i j m} \frac{\partial}{\partial x_{l}}, \quad i, k=1,2,3 ; \\
& A_{i+3, k+3}\left(\partial_{x}\right) \equiv c_{j i l k}^{\prime} \frac{\partial^{2}}{\partial x_{j} \partial x_{l}}-c_{j m l p} \varepsilon_{i j m} \varepsilon_{k l p}, \quad i, k=1,2,3 .
\end{aligned}
$$


Denote by $U=\left(U_{1}, \ldots, U_{6}\right)$ the six-component vector $U_{i}=u_{i}$ and $U_{i+3}=$ $\omega_{i}(i=1,2,3)$. Then the system (1) is written in the matrix form

$$
A\left(\partial_{x}\right) U=0 \quad\left(A_{i k}\left(\partial_{x}\right) U_{k}=0\right) .
$$

2. Let us establish the properties of the fundamental matrix $\Phi=\left\|\Phi_{i j}\right\|_{6 \times 6}$ of the operator $A\left(\partial_{x}\right)$ in the neighborhood of the point at infinity. By virtue of the definition of the fundamental matrix we have

$$
A_{i k}\left(\partial_{x}\right) \Phi_{k j}(x)=\delta_{i j} \delta(x), \quad i, j=1, \ldots, 6,
$$

where $\delta_{i j}$ is the Kronecker symbol and $\delta$ is the Dirac function. Using the Fourier transform

$$
\widehat{\varphi}(\xi) \equiv F[\varphi](\xi)=\int_{\mathbb{R}^{3}} e^{i x \cdot \xi} \varphi(x) d x,
$$

from this equality we obtain

$$
\begin{gathered}
-A_{i k}(\xi) \widehat{\Phi}_{k j}(\xi)=\delta_{i j}, \\
i, j=1, \ldots 6
\end{gathered}
$$

where

$$
\begin{gathered}
A(\xi) \equiv\left\|A_{i k}(\xi)\right\|_{6 \times 6}, \\
A_{i k}(\xi)=c_{j i l k} \xi_{j} \xi_{l}, \quad A_{i, k+3}(\xi)=i c_{j i l m} \varepsilon_{k l m} \xi_{j}, \\
A_{i+3, k}(\xi)=-i c_{j m l k} \varepsilon_{i j m} \xi_{l}, \\
A_{i+3, k+3}(\xi)=c_{j i l k}^{\prime} \xi_{j} \xi_{l}+c_{j m l p} \varepsilon_{i j m} \varepsilon_{k l p}, \\
i, k=1,2,3 .
\end{gathered}
$$

The matrix $A(\xi)$ is the invertible one if $|\xi| \equiv\left(\xi_{i} \xi_{i}\right)^{1 / 2} \neq 0$. Indeed, if $|\xi| \neq 0$ and $\eta_{i} \equiv \xi_{i} /|\xi|$, then

$$
\begin{aligned}
& \operatorname{det} A(\xi)=|\xi|^{6} \operatorname{det} B(\eta,|\xi|), \\
& B(\eta, \rho) \equiv\left\|B_{i k}(\eta, \rho)\right\|_{6 \times 6}, \\
& B_{i k}(\eta, \rho)=A_{i k}(\eta), \quad i \leq 3 \text { or } k \leq 3 ; \\
& B_{i+3, k+3}(\eta, \rho)=\rho^{2} c_{j i l k}^{\prime} \eta_{j} \eta_{l}+c_{j m l p} \varepsilon_{i j m} \varepsilon_{k l p}, \quad i \leq 3, \quad k \leq 3 .
\end{aligned}
$$

Now we will prove that $\operatorname{det} B(\eta, \rho) \neq 0$ for $\eta \neq 0$. Consider the expression

$$
B_{i k}(\eta, \rho) U_{i} U_{k}=c_{j i l k} \eta_{j} \eta_{l} u_{i} u_{k}+\rho^{2} c_{j i l k}^{\prime} \eta_{j} \eta_{l} \omega_{i} \omega_{k}+c_{j m l p} \varepsilon_{i j m} \varepsilon_{k l p} \omega_{i} \omega_{k} .
$$

By virtue of (3) we have the estimates

$$
\begin{aligned}
& c_{j i l k} \eta_{j} \eta_{l} u_{i} u_{k} \geq c_{0}\left(\eta_{j} u_{i}\right)\left(\eta_{j} u_{i}\right)=|\eta|^{2} c_{o} u_{i} u_{i}, \\
& c_{j i l k}^{\prime} \eta_{j} \eta_{l} \omega_{i} \omega_{k} \geq c_{0}\left(\eta_{j} \omega_{i}\right)\left(\eta_{j} \omega_{i}\right)=|\eta|^{2} c_{o} \omega_{i} \omega_{i}, \\
& c_{j m l p} \varepsilon_{i j m} \varepsilon_{k l p} \omega_{i} \omega_{k} \geq c_{0}\left(\varepsilon_{i j m} \omega_{i}\right)\left(\varepsilon_{k j m} \omega_{k}\right)=2 c_{o} \omega_{i} \omega_{i}
\end{aligned}
$$


for some positive number $c_{0}$. Therefore

$$
B_{i k}(\eta, \rho) U_{i} U_{k} \geq\left|\eta^{2}\right| c_{0} u_{i} u_{i}+|\eta|^{2} \rho^{2} c_{0} \omega_{i} \omega_{i}+2 c_{0} \omega_{i} \omega_{i}>0
$$

if $U \neq 0$ and $\eta \neq 0$. Therefore $\operatorname{det} B(\eta, \rho) \neq 0$ for $\eta \neq 0$.

Let us represent $\operatorname{det} B(\eta, r)$ as follows:

$$
\operatorname{det} B(\eta, \rho)=\sum_{k=0}^{6} a_{k}(\eta) \rho^{k},
$$

where $a_{k}(\eta)$ are homogeneous polynomials of $\eta$ of order $k+6$. In particular,

$$
a_{0}(\eta)=\operatorname{det} B(\eta, 0) \text {. }
$$

As proved above,

$$
a_{0}(\eta) \neq 0, \quad \sum_{k=0}^{6} a_{k}(\eta) \rho^{k} \neq 0 \quad \text { for } \eta \neq 0 .
$$

Write $\operatorname{det} A(\xi)$ in the form

$$
\operatorname{det} A(\xi)=|\xi|^{6} \sum_{k=0}^{6} a_{k}(\eta)|\xi|^{k} .
$$

By virtue of (7) $A(\xi)$ is the invertible matrix for $|\xi| \neq 0$. Therefore

$$
\widehat{\Phi}_{i k}(\xi)=-A_{i k}^{-1}(\xi), \quad i, k=1, \ldots, 6 .
$$

Let us now estimate the elements of the matrix $\widehat{\Phi}(\xi)$. First we will prove the validity of the representation

$$
\begin{gathered}
\widehat{\Phi}_{i k}(\xi)=\widehat{\Phi}_{i k}^{(1)}(\xi)+\widehat{\Phi}_{i k}^{(2)}(\xi), \\
i, k=1, \ldots, 6,
\end{gathered}
$$

where $\widehat{\Phi}_{i k}^{(1)}(\xi)$ are homogeneous functions of order -2 for $i, k=1,2,3$, of order -1 for $i=1,2,3$ and $k=4,5,6$, and for $i=4,5,6$ and $k=1,2,3$; of order 0 for $i, k=4,5,6 ; \widehat{\Phi}_{i k}^{(2)}(\xi)$ admits the estimates

$$
\begin{aligned}
& \left|\partial^{\alpha} \widehat{\Phi}_{i k}^{(2)}(\xi)\right| \leq c_{\alpha}|\xi|^{-|\alpha|-1}, \quad i \leq 3, k \leq 3 \\
& \left|\partial^{\alpha} \widehat{\Phi}_{i k}^{(2)}(\xi)\right| \leq c_{\alpha}|\xi|^{-|\alpha|}, \quad i \leq 3, k \geq 4 \text { or } i \geq 4, k \leq 3 \\
& \left|\partial^{\alpha} \widehat{\Phi}_{i k}^{(2)}(\xi)\right| \leq c_{\alpha}|\xi|^{1-|\alpha|}, \quad i \geq 4, k \geq 4
\end{aligned}
$$

$|\xi| \neq 0, \alpha$ is an arbitrary multiindex, and $c_{\alpha}=$ const. 
Let $F \equiv\left(F_{1}, \ldots, F_{6}\right)$ be some vector and $V_{i} \equiv A_{i k}^{-1} F_{k}$. Repeating the above arguments for the matrix $B(\eta, r)$, we can readily prove

$$
A_{i k}(\xi) V_{i} V_{k} \geq c_{0}|\xi|^{2} V_{i} V_{i}+2 c_{0} \sum_{i=1}^{4} V_{i}^{2} .
$$

Let us fix the index $p$. If $F_{k}=\delta_{k p}, k=1, \ldots, 6$, then $V_{i}=A_{i k}^{-1}(\xi) \delta_{k p}=$ $A_{i p}^{-1}(\xi)(i=1, \ldots, 6)$. The substitution of the obtained value of $V_{i}$ in (11) leads to

$$
A_{p p}^{-1}(\xi) \geq c_{0}|\xi|^{2} \sum_{k=1}^{6}\left(A_{k p}^{-1}(\xi)\right)^{2}+2 c_{0} \sum_{k=4}^{6}\left(A_{k p}^{-1}(\xi)\right)^{2}
$$

Hence

$$
\begin{gathered}
\sum_{k, p=1}^{6}\left(A_{k p}^{-1}(\xi)\right)^{2} \leq \frac{1}{c_{0}|\xi|^{2}} \sum_{p=1}^{6} A_{p p}^{-1}(\xi) \leq \frac{c_{1}}{|\xi|^{2}}\left(\sum_{k, p=1}^{6}\left(A_{k p}^{-1}(\xi)\right)^{2}\right)^{1 / 2}, \\
\left|A_{k p}^{-1}(\xi)\right| \leq c_{1}|\xi|^{-2}, \quad c_{1}=\text { const }, k, p=1, \ldots, 6 .
\end{gathered}
$$

From (12) and (13) we obtain

$$
\begin{gathered}
\sum_{k=4}^{6}\left(A_{k p}^{-1}(\xi)\right)^{2} \leq \frac{1}{2 c_{0}} A_{p p}^{-1}(\xi) \leq \frac{c_{1}}{2 c_{0}}|\xi|^{-2}, \\
\left|A_{k p}^{-1}(\xi)\right| \leq c_{2}|\xi|^{-1}, \quad k=4,5,6 ; p=1, \ldots, 6 .
\end{gathered}
$$

Since $A_{i k}(\xi)=A_{k i}(-\xi)(i, k=1, \ldots, 6)$, from (14) we have

$$
\left|A_{k p}^{-1}(\xi)\right| \leq c_{2}|\xi|^{-1}, \quad c_{1}=\text { const }, k=1, \ldots, 6 ; p=4,5,6 .
$$

Considering (12) for $p \geq 4$, we obtain

$$
\sum_{k, p=4}^{6}\left(A_{k p}^{-1}(\xi)\right)^{2} \leq \frac{1}{2 c_{0}} \sum_{p=4}^{6} A_{p p}^{-1}(\xi) \leq c_{1}\left(\sum_{k, p=4}^{6}\left(A_{k p}^{-1}(\xi)\right)^{2}\right)^{1 / 2} .
$$

Therefore

$$
\left|A_{k p}^{-1}(\xi)\right| \leq c_{1}, \quad k, p=4,5,6
$$

Now we will prove the representation (9). Let $i \leq 3$ and $k \leq 3$. Write $\widehat{\Phi}_{i k}(\xi)$ in the form

$$
\widehat{\Phi}_{i k}(\xi)=-A_{i k}^{-1}(i \xi)=-\frac{M_{i k}(\xi)}{\operatorname{det} A(\xi)},
$$


where $M_{i k}(\xi)$ is the cofactor of the element $A_{i k}(\xi)$ in the matrix $A(\xi)$. Therefore $M_{i k}(\xi)$ is the polynomial of $\xi$. Since $\operatorname{det} A(\xi)=|\xi|^{6} \operatorname{det} B(\eta,|\xi|)$ and $\left|\widehat{\Phi}_{i k}(\xi)\right| \leq c|\xi|^{-2}$, it is obvious that $M_{i k}(\xi)$ is represented in the form

$$
M_{i k}(\xi)=|\xi|^{4} \sum_{j=0}^{6} b_{j}^{i k}(\eta)|\xi|^{j},
$$

where $b_{j}(\eta)$ is the homogeneous polynomial of $\eta$ of order $j+4(j=1, \ldots, 6)$. Thus

$$
\widehat{\Phi}_{i k}(\xi)=-\frac{1}{|\xi|^{2}} \frac{\sum_{j=0}^{6} b_{j}^{i k}(\eta)|\xi|^{j}}{\sum_{j=0}^{6} a_{j}(\eta)|\xi|^{j}} .
$$

Setting

$$
\begin{aligned}
& \widehat{\Phi}_{i k}^{(1)}(\xi)=-\frac{1}{|\xi|^{2}} \cdot \frac{b_{0}^{i k}(\eta)}{a_{0}(\eta)}=-\frac{1}{|\xi|^{2}} \frac{b_{0}^{i k}\left(\frac{\xi}{|\xi|}\right)}{a_{0}\left(\frac{\xi}{|\xi|}\right)}, \\
& \widehat{\Phi}_{i k}^{(2)}(\xi)=-\frac{1}{|\xi|} \frac{\sum_{j=1}^{6}\left(a_{0}(\eta) b_{j}^{i k}(\eta)-b_{0}^{i k}(\eta) a_{j}(\eta)\right)|\xi|^{j-1}}{a_{0}(\eta) \sum_{j=0}^{6} a_{j}(\eta)|\xi|^{j}},
\end{aligned}
$$

we obtain the required representation $(9)$, since $\widehat{\Phi}_{i k}^{(1)}(\xi)$ is a homogeneous function of $\xi$ of order -2 and $\widehat{\Phi}_{i k}^{(2)}(\xi)$ satisfies the condition (10) for $i, k=$ $1,2,3$.

In a similar manner one can prove the validity of the representation (9) for the rest of $i$ and $k$.

Let us now estimate the matrix $\Phi(x)$. From the equality (9) we have

$$
\Phi_{i k}(x)=\Phi_{i k}^{(1)}(x)+\Phi_{i k}^{(2)}(x), \quad i, k=1, \ldots, 6 .
$$

The first term in (17) is the inverse Fourier transform of the homogeneous function $\widehat{\Phi}_{i k}^{(1)}(\xi)$, and therefore $\Phi_{i k}^{(1)}(\xi)$ is a homogeneous function of order $-3-q$, where $q$ is the order of the homogeneous function $\widehat{\Phi}_{i k}^{(1)}(\xi)$. Thus for $\Phi_{i k}^{(1)}(\xi)$ we have the estimates

$$
\begin{aligned}
& \left|\partial^{\alpha} \Phi_{i k}^{(1)}(x)\right| \leq c|x|^{-|\alpha|-1}, \quad i \leq 3, \quad k \leq 3 ; \\
& \left|\partial^{\alpha} \Phi_{i k}^{(1)}(x)\right| \leq c|x|^{-|\alpha|-2}, \quad i \leq 3, \quad k \geq 4 \text { or } i \geq 3, \quad k \leq 4 \\
& \left|\partial^{\alpha} \Phi_{i k}^{(1)}(x)\right| \leq c|x|^{-|\alpha|-3}, \quad i, k \geq 4, \quad c=\text { const. }
\end{aligned}
$$


Next we will estimate the second term in (17). It will be shown that in the neighborhood of the point at infinity

$$
\begin{aligned}
& \partial^{\alpha} \Phi_{i k}^{(2)}(x)=o\left(|x|^{-|\alpha|-1}\right), \quad i \leq 3, \quad k \leq 3 \\
& \partial^{\alpha} \Phi_{i k}^{(2)}(x)=o\left(|x|^{-|\alpha|-2}\right), \quad i \leq 3, \quad k \geq 4 \quad \text { or } \quad i \geq 3, \quad k \leq 4 \\
& \partial^{\alpha} \Phi_{i k}^{(2)}(x)=o\left(|x|^{-|\alpha|-3}\right), \quad i \geq 4, \quad k \geq 4 .
\end{aligned}
$$

We introduce the functions $\omega_{0}$ and $\omega_{1}$, where $\omega_{1}=1-\omega_{0}$ and $\omega_{0}$ possesses the following properties:

$$
\omega_{0} \in C^{\infty}\left(\mathbb{R}^{3}\right), \quad \operatorname{supp} \omega_{0} \subset B(0,1), \quad \omega_{0}(x)=1 \text { if }|x| \leq \frac{1}{2} .
$$

Here $B(0,1)$ is the ball with center 0 and radius 1 in $\mathbb{R}^{3}$. Obviously,

$$
\begin{aligned}
\widehat{\Phi}_{i k}^{(2)}(\xi) & =\widehat{\Phi}_{i k}^{(2)}(\xi) \omega_{0}(\xi)+\widehat{\Phi}_{i k}^{(2)}(\xi) \omega_{1}(\xi), \\
\Phi_{i k}^{(2)}(x) & ={ }^{0}(2)(x)+\stackrel{1}{\Phi}_{i k}^{(2)}(x),
\end{aligned}
$$

where

$$
\stackrel{0}{\Phi}_{i k}^{(2)}(x)=F^{-1}\left[\widehat{\Phi}_{i k}^{(2)} \omega_{0}\right](x), \quad \stackrel{1}{\Phi}_{i k}^{(2)}(x)=F^{-1}\left[\widehat{\Phi}_{i k}^{(2)} \omega_{1}\right](x) .
$$

$F^{-1}$ is the inverse Fourier transform operator.

Let $i \leq 3, k \leq 3$ and $|\beta|<\alpha+2$. Then by virtue of (10) the function $\partial^{\beta}\left(\xi^{\alpha} \widehat{\Phi}_{i k}^{(2)}(\xi) \omega_{0}(\xi)\right)$ is absolutely integrable on $\mathbb{R}^{3}$ and therefore the inverse Fourier transform of this function tends to zero at infinity, but

$$
F^{-1}\left[\partial^{\beta}\left(\xi^{\alpha} \widehat{\Phi}_{i k}^{(2)}(\xi) \omega_{0}(\xi)\right)\right](x)=(-1)^{|\alpha|} i^{|\alpha|+|\beta|} x^{\beta} \partial^{\alpha} \stackrel{0}{\Phi}_{i k}^{(2)}(x) .
$$

Thus, if $|\beta|=|\alpha|+1$, then

$$
\lim _{|x| \rightarrow \infty} x^{\beta} \partial^{\alpha} \stackrel{0}{\Phi_{i k}^{(2)}}(x)=0
$$

and therefore

$$
\partial^{\alpha} \stackrel{0}{\Phi_{i k}^{(2)}}(x)=o\left(|x|^{-|\alpha|-1}\right) .
$$

Let us estimate ${ }^{1}(2)(x)$. If $n \geq|\alpha|+2$, then

$$
\Delta^{n}\left(\xi^{\alpha} \widehat{\Phi}_{i k}^{(2)}(\xi) \omega_{1}(\xi)\right) \in L_{1}\left(\mathbb{R}^{3}\right)
$$

and the Fourier transform of this expression tends to zero at infinity:

$$
(-1)^{n}|x|^{2 n} \partial^{\alpha} \stackrel{1}{\Phi_{i k}^{(2)}}(x)=o(1) .
$$


Therefore for any $n \geq|\alpha|+2$

$$
\partial^{\alpha} \stackrel{1}{\Phi}_{i k}^{(2)}(x)=o\left(|x|^{-2 n}\right)
$$

Equations (20) and (21) imply the first estimate in (19). The rest of the estimates are proved in the same manner.

3. The derivation of the asymptotic representation formula for the solution of the system (1) in the neighborhood of the point at infinity is based on the Green formulas. We will give these formulas.

Let $\Omega$ be a bounded domain in $\mathbb{R}^{3}$ with a piecewise-smooth boundary $\partial \Omega, U=\left(U_{1}, \ldots, U_{6}\right), V=\left(V_{1}, \ldots, V_{6}\right), U \in C^{2}(\bar{\Omega})$ and $V \in C^{2}(\bar{\Omega})$. Then

$$
\begin{gathered}
\int_{\Omega}\left(V_{i}(x) A_{i k} U_{k}(x)-U_{k}(x) A_{k i}\left(\partial_{x}\right) V_{i}(x)\right) d x= \\
=\int_{\partial \Omega}\left(V_{i}(y) T_{i k}\left(\partial_{y}, \nu\right) U_{k}(y)-U_{k}(y) T_{k i}\left(\partial_{y}, \nu\right) V_{i}(y)\right) d y S,
\end{gathered}
$$

where $T\left(\partial_{y}, \nu\right) \equiv\left\|T_{i k}\left(\partial_{y}\right)\right\|_{6 \times 6}$ is the boundary stress operator defined on $\partial \Omega$ by the relations

$$
\begin{aligned}
& T_{i k}\left(\partial_{y}, \nu\right)=c_{j i l k} \nu_{j} \frac{\partial}{\partial y_{l}} \\
& T_{i, k+3}\left(\partial_{y}, \nu\right)=-c_{j i l m} \nu_{j} \varepsilon_{k l m} \\
& T_{i+3, k}\left(\partial_{y}, \nu\right)=0 \\
& T_{i+3, k+3}\left(\partial_{y}, \nu\right)=c_{j i l k}^{\prime} \nu_{j} \frac{\partial}{\partial y_{l}}, \quad i, k=1,2,3 .
\end{aligned}
$$

Here $\nu=\left(\nu_{1}, \nu_{2}, \nu_{3}\right)$ is the unit normal to $\partial \Omega$ at the point $y$, external with respect to $\Omega$.

If $U=\left(U_{1}, \ldots, U_{6}\right)$ is the solution of the system (1) in the domain $\Omega$, belonging to the class $C^{2}(\Omega) \cap C^{1}(\bar{\Omega})$, then $\forall x \in \Omega$ :

$$
\begin{gathered}
u_{j}(x)=\int_{\partial \Omega}\left(U_{i}(y) T_{i k}\left(\partial_{y}, \nu\right) \Phi_{k j}(y-x)-\right. \\
\left.-\Phi_{k j}(y-x) T_{k i}\left(\partial_{y}, \nu\right) U_{i}(y)\right) d y S
\end{gathered}
$$

The formulas (22) and (24) are proved by the standard techniques [2], $[6],[8]$. 
4. Let us formulate the theorem of the asymptotic representation of a solution of the system (1) in the neighborhood of the point at infinity.

Theorem 1. Let $\Omega$ be a domain from $\mathbb{R}^{3}$ containing the neighborhood of the point at infinity, let $U$ be a solution of the system (1) in $\Omega$ of the class $C^{2}(\Omega)$, and let

$$
U_{i}(z)=o\left(|z|^{p+1}\right), \quad i=1, \ldots, 6
$$

in the neighborhood of the point at infinity, where $p$ is a nonnegative integer number. Then the representation

$$
\begin{aligned}
& U_{j}(x)=\sum_{|\alpha| \leq p} c_{j}^{(\alpha)} x^{\alpha}+\sum_{|\beta| \leq q} d_{k}^{(\beta)} \partial^{\beta} \Phi_{j k}(x)+\psi_{j}(x) \\
& j=1, \ldots, 6
\end{aligned}
$$

holds in the neigborhood of the point at infinity. Here $c_{j}^{(\alpha)}$ and $d_{k}^{(\beta)}$ are the constants, $\alpha=\left(\alpha_{1}, \alpha_{2}, \alpha_{3}\right)$ and $\beta=\left(\beta_{1}, \beta_{2}, \beta_{3}\right)$ are the multiindexes, $q$ is an arbitrary nonnegative integer number, and the function $\psi_{j}$ admits the estimate

$$
\partial^{\gamma} \psi_{j}(x)=O\left(|x|^{-2-|\gamma|-q}\right), \quad j=1, \ldots, 6
$$

in the neighborhood of $|x|=\infty$, where $\gamma \equiv\left(\gamma_{1}, \gamma_{2}, \gamma_{3}\right)$ is an arbitrary multiindex.

Moreover, each of the three terms on the right-hand side of (26) is the solution of the system (1) in the neighborhood of $|x|=\infty$.

Proof. Let $x \in \Omega$, and let a positive number $r$ be chosen such that $x \in B(0, r / 8)$ and $\mathbb{R}^{3} \backslash B(0, r / 8) \subset \Omega$. Write the formula (24) for the domain $\Omega_{r} \equiv B(0, r) \cap \Omega$. We will have

$$
\begin{gathered}
U_{j}(x)=\int_{\partial \Omega}\left(U_{i}(y) T_{i k}\left(\partial_{y}, \nu\right) \Phi_{k j}(y-x)-\right. \\
\left.-\Phi_{k j}(y-x) T_{k i}\left(\partial_{y}, \nu\right) U_{i}(y)\right) d_{y} S+ \\
+\int_{\partial B(0, r)}\left(U_{i}(y) T_{i k}\left(\partial_{y}, \nu\right) \Phi_{k j}(y-x)-\right. \\
\left.-\Phi_{k j}(y-x) T_{k i}\left(\partial_{y}, \nu\right) U_{i}(y)\right) d_{y} S .
\end{gathered}
$$


Represent $\Phi_{k j}(y-x)$, in the neighborhood of the point $y$, by the Taylor's formula

$$
\begin{gathered}
\Phi_{k j}(y-x)=\sum_{|\alpha| \leq p+1} \frac{(-1)^{|\alpha|} x^{\alpha}}{\alpha !} \partial^{\alpha} \Phi_{k j}(y)+R_{k j}(x, y), \\
R_{k j}(x, y)=\sum_{|\alpha| \leq p+2} \frac{(-1)^{|\alpha|} x^{\alpha}}{\alpha !} \partial^{\alpha} \Phi_{k j}(y-\theta x), \quad 0<\theta<1 .
\end{gathered}
$$

By virtue of (18) and (19) we readily ascertain that the estimates

$$
\begin{array}{ll}
\left|\partial_{y}^{\beta} R_{k j}(x, y)\right| \leq a^{\beta, p}(r)|y|^{-p-|\beta|-3}, & k, j \leq 3 \\
\left|\partial_{y}^{\beta} R_{k j}(x, y)\right| \leq a^{\beta, p}(r)|y|^{-p-|\beta|-4}, & k \leq 3, j \geq 4 \text { or } k \geq 4, j \leq 3 \\
\left|\partial_{y}^{\beta} R_{k j}(x, y)\right| \leq a^{\beta, p}(r)|y|^{-p-|\beta|-4}, & k, j \geq 4
\end{array}
$$

are fulfilled for any $x$ and $y$ satisfying the conditions $|x|<r / 8$ and $r / 4 \leq$ $|y| \leq r$. Taking into account (29), from (28) we obtain

$$
\begin{gathered}
U_{j}(x)=U_{j}^{(0)}(x)+\sum_{\substack{|\alpha| \leq p+1 \\
j=1, \ldots, 6,}} \frac{(-1)^{|\alpha|} c_{j}^{(\alpha)}(r)}{\alpha !} x^{\alpha}+I_{j}(p, r, x), \\
\text {, }
\end{gathered}
$$

where

$$
\begin{gathered}
U_{j}^{(0)}(x) \equiv \int_{\partial \Omega}\left(U_{i}(y) T_{i k}\left(\partial_{y}, \nu\right) \Phi_{k j}(y-x)-\right. \\
\left.-\Phi_{k j}(y-x) T_{k i}\left(\partial_{y}, \nu\right) U_{i}(y)\right) d_{y} S, \\
c_{j}^{(\alpha)}(r) \equiv \int_{\partial \Omega}\left(U_{i}(y) T_{i k}\left(\partial_{y}, \nu\right) \partial^{\alpha} \Phi_{k j}(y)-\right. \\
\left.-\partial^{\alpha} \Phi_{k j}(y) T_{k i}\left(\partial_{y}, \nu\right) U_{i}(y)\right) d_{y} S, \\
I_{j}(p, r, x) \equiv \int_{\partial B(0, r)}\left(U_{i}(y) T_{i k}\left(\partial_{y}, \nu\right) R_{k j}(x, y)-\right. \\
\left.-R_{k j}(x, y) T_{k i}\left(\partial_{y}, \nu\right) U_{i}(y)\right) d_{y} S .
\end{gathered}
$$

It is not difficult to prove (cf. [6]) that $c_{j}^{(\alpha)}(r)$ does not depend on $r$, and, introducing the notation

$$
c_{j}^{(\alpha)} \equiv \frac{(-1)^{|\alpha|}}{\alpha !} c_{j}^{(\alpha)}(r)
$$

we obtain the equality

$$
U_{j}(x)=U_{j}^{(0)}(x)+\sum_{|\alpha| \leq p+1} c_{j}^{(\alpha)} x^{\alpha}+I_{j}(p, r, x),
$$


from which we conclude that $I_{j}(p, r, x)$ does not depend on $r$ either. Thus, if we prove

we will obtain

$$
\lim _{r \rightarrow \infty} I_{j}(p, r, x)=0,
$$

$$
U_{j}(x)=U_{j}^{(0)}(x)+\sum_{|\alpha| \leq p+1} c_{j}^{(\alpha)} x^{\alpha} .
$$

Let the function $\omega: \mathbb{R}^{3} \rightarrow \mathbb{R}, \omega \in C_{0}^{\infty}\left(\mathbb{R}^{3}\right)$, supp $\omega \subset B(0,3) \backslash B(0,1 / 3)$, $\omega(y)=1$ for $1 / 2<|y|<2$. Then the estimate

$$
\left|\partial^{\alpha} \omega^{(r)}(y)\right| \leq b^{(\alpha)} r^{-|\alpha|}
$$

holds for the function $\omega^{(r)}(y) \equiv \omega(y / r)$.

Rewriting the formula (22) for the domain $B(0, r) \backslash B(0, r / 4)$, in which $V$ is replaced by the function

$$
\begin{gathered}
V \equiv\left(R_{1 j}^{(r)}(x, \cdot), \ldots, R_{6 j}^{(r)}(x, \cdot)\right), \\
R_{k j}^{(r)}(x, y) \equiv \omega^{(r)}(y) R_{k j}(x, y),
\end{gathered}
$$

we obtain

$$
\begin{gathered}
I_{j}(p, r, x)=\int_{\begin{array}{c}
B(0, r) \backslash B(0, r / 4) \\
j=1, \ldots, 6 .
\end{array}} U_{i}(z) A_{i k}\left(\partial_{z}\right) R_{k j}^{(r)}(x, z) d z,
\end{gathered}
$$

On account of (30) we have the estimates

$$
\begin{array}{ll}
\left|A_{i k}\left(\partial_{z}\right) R_{k j}(x, z)\right| \leq a(x)|z|^{-p-5}, & i \leq 3, j \leq 3 ; \\
\left|A_{i k}\left(\partial_{z}\right) R_{k j}(x, z)\right| \leq a(x)|z|^{-p-6}, & i \leq 3, j \geq 4 ; \\
\left|A_{i k}\left(\partial_{z}\right) R_{k j}(x, z)\right| \leq a(x)|z|^{-p-4}, & i \geq 4, j \leq 3 ; \\
\left|A_{i k}\left(\partial_{z}\right) R_{k j}(x, z)\right| \leq a(x)|z|^{-p-5}, & i \geq 4, j \geq 4 .
\end{array}
$$

Taking these estimates and restrictions (25) into account, we obtain

$$
\lim _{r \rightarrow \infty} I_{j}(p, r, x)=0 .
$$

The representation (35) is thus derived. Note that, due to (25), in the formula (35) the constants $c_{j}^{(\alpha)}=0$ if $\alpha=p+1$, and therefore we have the representation

$$
U_{j}(x)=U_{j}^{(0)}(x)+\sum_{|\alpha| \leq p} c_{j}^{(\alpha)} x^{\alpha} .
$$

Let us transform this representation in the form (26). To this effect, in the formula (32) we will represent $\Phi_{k j}(y-x)$ by the Taylor's formula. Since $A_{k j}(\xi)=A_{j k}(-\xi)$, we have $A_{k j}^{-1}(\xi)=A_{j k}^{-1}(-\xi)$, and therefore $\Phi_{k j}(y-x)=$ 
$\Phi_{j k}(x-y)$. Choose a positive number $r_{0}$ such that $\mathbb{R}^{3} \backslash B\left(0, r_{0}\right) \subset \Omega$. Then, if $y \in \partial \Omega$ and $x \in \mathbb{R}^{3} \backslash B\left(0,2 r_{0}\right)$, we will have the expansion

$$
\begin{gathered}
\Phi_{k j}(y-x)=\Phi_{j k}(x-y)= \\
=\sum_{|\alpha| \leq q} \frac{(-1)^{|\alpha|} y^{\alpha}}{\alpha !}\left(\partial^{\alpha} \Phi_{j k}\right)(x)+\psi_{j k}(x, y), \\
\psi_{k j}(x, y)=\sum_{|\alpha|=q+1} \frac{(-1)^{q+1} y^{\alpha}}{\alpha !}\left(\partial^{\alpha} \Phi_{j k}\right)(x-\theta y), \\
0<\theta<1 .
\end{gathered}
$$

Applying the estimates (18), (19), we show that

$$
\begin{gathered}
\left|\partial_{x}^{\beta} \psi_{j k}(x, y)\right| \leq c_{j k}^{(\beta)}(y)|x|^{-q-|\beta|-2}, \\
j \leq 3, \quad k \leq 3 \\
\left|\partial_{x}^{\beta} \psi_{j k}(x, y)\right| \leq c_{j k}^{(\beta)}(y)|x|^{-q-|\beta|-3}, \\
j \leq 3, \quad k \geq 4 \text { or } j \geq 4, \quad k \leq 3 ; \\
\left|\partial_{x}^{\beta} \psi_{j k}(x, y)\right| \leq c_{j k}^{(\beta)}(y)|x|^{-q-\beta-4}, \\
j \geq 4, \quad k \geq 4 .
\end{gathered}
$$

The substitution of (38) in (32) gives

$$
\begin{gathered}
U_{j}^{(0)}(x)=\sum_{|\alpha| \leq q} d_{k}^{(\alpha)} \partial^{\alpha} \Phi_{j k}(x)+\psi_{j}(x), \\
\psi_{j}(x)=(-1)^{q} \sum_{|\alpha|=q} \int_{\partial \Omega} U_{i}(y) \frac{y^{\alpha}}{\alpha !} T_{i k}(\partial x, \nu)\left(\partial^{\alpha} \Phi_{j k}\right)(x) d y S- \\
-\int_{\partial \Omega}\left(U_{i}(y) T_{i k}(\partial x, \nu) \psi_{j k}(x, y)-\psi_{j k}(x, y) T_{k i}(\partial y, \nu) U_{i}(y)\right) d y S .
\end{gathered}
$$

Now, due to (18), (19) and (39), we obtain

$$
\left|\partial^{\gamma} \psi_{j}(x)\right| \leq c_{j}^{(\gamma)}|x|^{-|\gamma|-2-q}, \quad j=1, \ldots, 6 .
$$

Remark. Theorem 1 can also be proved when the condition (25) is replaced by the conditions of Theorem 2 from [6].

5. Theorem 1 can be used, in particular, to prove uniqueness theorems for the external boundary value problems of the couple-stress theory of elasticity, and to weaken the restrictions imposed on the class of solutions. As an example, let us consider the first external problem: 
In the domain $\Omega^{-}$with the piecewise-smooth boundary $\partial \Omega$, find a solution $U$ of the system (1) of the class $C^{1}(\bar{\Omega}) \cap C^{2}(\Omega)$, satisfying the boundary condition

$$
\forall y \in \partial \Omega: \lim _{\Omega^{-} \ni x \rightarrow y} U(x)=\varphi(y)
$$

and the condition at infinity

$$
\lim _{|x| \rightarrow \infty} U(x)=0 .
$$

Theorem 2. The first external problem of the couple-stress theory of elasticity has at most one solution.

Proof. Let $U$ be a solution of the first external problem. Then the expansion (26) holds for $U$. Setting $p=0, q=0$ in (26), we obtain the equality

$$
U_{j}(x)=c_{j}^{(0)}+d_{k}^{(0)} \Phi_{j k}(x)+\psi_{j}(x), \quad j=1, \ldots, 6 .
$$

All terms on the right-hand side of this equality, except $c_{j}^{(0)}$, tend to zero as $|x| \rightarrow \infty$. Therefore $c_{j}^{(0)}=0, j=1, \ldots, 6$. Now we conclude from (18), (19), (27) that

$$
\begin{array}{ll}
\partial^{\alpha} U_{j}(x)=O\left(|x|^{-|\alpha|-1}\right), & j=1,2,3 ; \\
\partial^{\alpha} U_{j}(x)=O\left(|x|^{-|\alpha|-2}\right), & j=4,5,6 .
\end{array}
$$

Now, repeating the arguments, say, from [2], we readily obtain the proof of Theorem 2.

The uniqueness theorems for the other external boundary value problems of the couple-stress elasticity are proved in a similar manner.

\section{REFERENCES}

1. R.J. Knops and L.E. Payne, Uniqueness theorems in linear elasticity. Springer tracts in natural philosophy, v. 19, Springer-Verlag, Berlin-Heidelberg-New York, 1971.

2. V.D. Kupradze, T.G. Gegelia, M.O. Basheleishvili, and T.V. Burchuladze, Three-dimensional problems of the mathematical theory of elasticity and thermoelasticity. (Translated from the Russian) North-Holland Series in Applied Mathematics and Mechanics, v. 25, Amsterdam, New York, Oxford, North-Holland Publishing Company, 1979.

3. T.V. Buchukuri and T.G. Gegelia, Stress and displacement behavior near a singular point. (Russian) Reports of extended sessions of the seminar of I.N. Vekua Inst. of appl. math. (Russian) 2(1986), No. 2, 25-28. 
4. V.A. Kondratiev and O.A. Oleinik, Korn's inequalities and the uniqueness of solutions of classical boundary value problems in unbounded domains for a system in elasticity theory. (Russian) Current problems in mathematical physics. (Russian) (Proceedings of the all-union symposium; Tbilisi, 1987), v. 1, 35-63, Tbilisi University Press, Tbilisi, 1987.

5. - - On the behavior at infinity of solutions of elliptic systems with a finite energy integral. Arch. rational Mech. Anal. 99(1987), No. 1, 75-89.

6. T.V. Buchukuri and T.G. Gegelia, Qualitative properties of solutions of the basic equations of the theory of elasticity near singular points. (Russian) Trudy Tbilissk. Mat. Inst. Razmadze 90(1988), 40-67.

7. - - On the uniqueness of solutions of the basic problems of elasticity for infinite domains. (Russian) Differentsial'nye Uravneniya 25(1988), No. 9, 1556-1565.

8. F. John, Plane waves and spherical means. Interscience Publishers, New York, 1955.

9. V. Nowacki, The theory of elasticity. (Translation from Polish into Russian) Mir, Moscow, 1975.

(Received 02.03.1993)

Authors' address:

A.Razmadze Mathematical Institute

Georgian Academy of Sciences

1, Z. Rukhadze St., 380093 Tbilisi

Republic of Georgia 